\title{
Determinación de Necesidades de Capacitación del Personal Administrativo del Área de Ciencias Económicas y de la Gestión de la UNMSM
}

\author{
Determination of Training Needs of the Administrative \\ Personnel of the Area of Economic Sciences and \\ Management of the UNMSM
}

\begin{abstract}
RESUMEN
Presentamos el avance del proceso de capacitación del personal administrativo de las facultades del área de ciencias económicas y de la gestión de la Universidad Nacional Mayor de San Marcos (UNMSM) en el marco de la implementación de la Ley del Servicio Civil. Se han desarrollado entrevistas a los funcionarios encargados de la gestión administrativa y aplicado un cuestionario al personal administrativo para determinar las necesidades de capacitación. Asimismo, se han estudiado los perfiles de puesto y perfiles personales para los cargos de administración de las referidas facultades. El resultado más resaltante es que se debe hacer hincapié en el desarrollo de habilidades blandas. Entre éstas destacan el trabajo en equipo y la resolución de conflictos. Asimismo, hemos hallado que, debido a la constante rotación de personal y a los bajos salarios, existe personal cada vez menos calificado lo cual es más notorio en el caso de personal contratado. En cuanto a la implementación de la Ley del Servicio Civil, las facultades estudiadas no han implementado ni planes ni presupuesto para la capacitación de su personal administrativo, lo cual pone en peligro la calidad del servicio que prestan. Si bien es cierto la UNMSM está en proceso de mejora se debe reforzar la capacitación del personal puesto que en este ámbito y en la aplicación de la Ley de Servicio Civil estamos todavía atrasados.
\end{abstract}

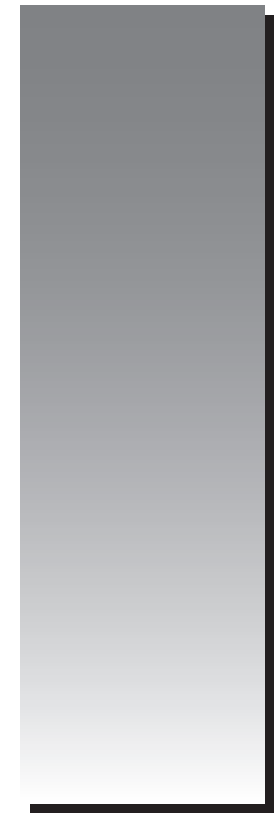

Walter David Ugarte Casafranca $^{1}$

wugartec@unmsm.edu.pe

Teresa Victoria Melgar Campos $^{1}$

teresa.melgar@unmsm.edu.pe

Elvis Fernando Tacillo Yauli $^{1}$

etacilloy@unmsm.edu.pe

Palabras clave: capacitación; servidor público y administrativo.

\begin{abstract}
We present the progress of the training process of the administrative staff of the faculties of the area of economic sciences and of the management of the UNMSM in the framework of the implementation of the Civil Service Law (SERVIR). Interviews have been conducted with the officials in charge of administrative management and a questionnaire has been applied to administrative staff to determine training needs. Likewise, the job profiles and personal profiles for the administration positions of the aforementioned faculties have been studied. The most outstanding result is that emphasis should be placed on the development of soft skills. These
\end{abstract}


include teamwork and conflict resolution. We have also found that, due to the constant turnover of staff and low salaries, there is less qualified personnel, which is more noticeable in the case of hired personnel. Regarding the implementation of the Civil Service Law, the faculties studied have not implemented any plans or budget for the training of their administrative staff, which jeopardizes the quality of the service they provide. Although it is true that UNMSM is in the process of improvement, training of personnel must be reinforced since in this area and in the application of the Civil Service Law (SERVIR) we are still behind.

Keywords: training; public servant and administrative.

\section{INTRODUCCIÓN}

\section{Situación problemática}

La gestión pública en el Perú requiere de trabajar en acciones de capacitación. La calidad de los servicios que presta el Estado es deficiente primordialmente por falta de capacitación del personal. La ausencia de personal calificado origina retrasos en las prestaciones estatales que se agudizan en la medida en que la demanda de la población es más grande. Si bien es cierto la UNMSM está en proceso de mejora se debe reforzar la capacitación del personal puesto que en este ámbito y en la aplicación de la Ley de Servicio Civil estamos todavía atrasados. Requerimos de funcionarios de carrera que sepan sustentar los proyectos propuestos por las autoridades y los órganos de gobierno universitarios. La UNMSM deberá trabajar en acciones de prospectiva en las que se deben resolver los problemas de capacitación y mejora de los servicios académicos y administrativos que presta a la comunidad universitaria y a la sociedad civil.

\section{Trabajos previos}

Segovia (2013) en su tesis para optar el grado de Magíster en Gestión y Política de la Innovación y la Tecnología en la Pontificia Universidad Católica del Perú, la gestión del conocimiento en una entidad pública a través del uso de plataformas virtuales de enseñanza: Caso Defensoría del Pueblo; indica que:

El modelo de gestión del conocimiento en la Defensoría del Pueblo está basado en dos pilares fundamentales que son la generación y transferencia del conocimiento, realizados a través de las acciones siguientes: la capacitación a través del uso de tecnologías de información y comunicación; y la educación priorizada en la capacitación a sus funcionarios. (p. 99)

Asimismo, concluye que "la capacitación de los funcionarios se da en dos momentos principales, primero durante la identificación de la necesidad de mejorar el desempeño del capital humano, y después por la necesidad de utilizar las tecnologías para la educación".

Alva (2014) en su tesis Las tecnologías de información y comunicación como instrumentos eficaces en la capacitación del personal: el caso de la Oficina Nacional de Procesos Electorales (ONPE) para optar el Grado Académico de Magíster en Administración con Mención en Dirección de Recursos Humanos en la Facultad de Ciencias Administrativas de la UNMSM sostiene que es necesario:

la capacitación encuentre fundamento en su contribución al desempeño de las organizaciones estatales para la realización del interés público, debe resultar funcional a una política de desarrollo de las competencias del personal. De allí que los proyectos de capacitación destinados a directivos, agentes y funcionarios públicos se orienten a mejorar sus competencias profesionales para la función, a la adquisición y fortalecimiento de las competencias básicas y específicas. (p. 3)

Medina (2014) en su tesis para la obtención del grado de Magíster en Dirección Estratégica de Recursos Humanos Diseño del plan de capacitación en base al modelo de retorno de inversión - ROI - para la planta central del 
ministerio de defensa nacional, correspondiente al periodo fiscal 2013 indica:

definir la pertinencia en los diferentes niveles de necesidades de capacitación y niveles de evaluación de programas de capacitación, facilitó la comprensión y colaboración de los diferentes miembros de la institución, quienes apoyaron la aprobación del Plan de Capacitación y la ejecución de los programas de capacitación, porque conocían la importancia que tenían los mismos en el desarrollo y la gestión institucional a través de su personal. (p. 79)

\section{Conceptos relacionados al tema}

\section{Capacitación.}

\section{Noción.}

Según Mustaffá (1976) la capacitación o desarrollo de personal es toda actividad realizada en una organización, respondiendo a sus necesidades, que busca mejorar la actitud, conocimiento, habilidades o conductas de su personal.

En este sentido, debido a las transformaciones en las actividades y estructuras organizacionales en las empresas, los cargos estáticos y fijos en la práctica casi no existen. Por ello, los colaboradores deben estar preparados para hacerse cargo de las funciones que requiera la organización. Este entorno siempre cambiante influye sobre los conocimientos y la ejecución de las tareas de cada uno de los integrantes.

En toda organización la capacitación cumple un objetivo importante y vital para su supervivencia. Es menester recordar que en toda entidad cada uno de sus elementos cumple una función para mantenerla en equilibrio. Reza (1995) dice:

el corazón hará circular la sangre llevando el oxígeno a todo el cuerpo, los riñones eliminarán las toxinas, el hígado fabricará las sustancias necesarias para la vida, etc., también en toda organización cada parte cumple con una misión. (p. 22)

El objetivo principal de la capacitación es el de potenciar las capacidades del personal para que mejoren la eficiencia en su trabajo. Sin embargo, no es responsable de que las acciones de los trabajadores sean erróneas o eficientes. Según Reza (1995) en esto consiste la función de cada trabajador, así como la de su supervisor. Siguiendo la idea, si las personas simplemente no tienen las condiciones anímicas ni las actitudes requeridas, ningún programa de adiestramiento podrá lograr que lo hagan adecuadamente; por ello, además de la capacitación, que es necesaria para potenciar las competencias laborales, requiere de la actitud de las personas para cumplir con su labor, es decir del compromiso que asuman con su trabajo.

Subrayamos entonces que la capacitación es un instrumento para aumentar la productividad; no es una finalidad organizacional. Salvo las instituciones educativas cuya misión es educar y su objetivo es el aprendizaje de sus beneficiarios. En el mundo empresarial, el saber hacer y hacerlo se privilegia al simple conocimiento. En resumen, la capacitación debe lograr que las personas potencien sus competencias para hacer bien lo que deben hacer bien.

\section{Planeación de la capacitación.}

Una empresa se constituye como un sistema y todos sus subsistemas tienen que marchar en un mismo sentido y en forma óptima para que la organización cumpla con sus propósitos.

Reza (1995) afirma que la planificación de la capacitación es establecer los procedimientos a realizar en tres etapas:

1. Detección de necesidades de capacitación

2. Programación y presupuesto

3. Establecimiento de objetivos

Detectar las necesidades de aprendizaje es el primer paso sine qua non de la planeación. Esto se cumple siguiendo cuatro pasos:

1. Ubicar las áreas que requieren capacitación para incrementar la productividad.

2. Identificar a los trabajadores que requieran capacitación específica.

3. Determinar el nivel y la medida suficiente de capacitación que necesita el empleado para que obtenga las competencias de su especialidad y esté preparado para ejecutarla. 
4. Determinar las prioridades y los medios financieros y humanos con que cuenta la empresa y el factor de beneficio de retorno que recibirá como consecuencia de la capacitación.

La empresa forma parte y al mismo tiempo se constituye en un sistema de interacción social integrada por personas que gestionan recursos de diferente índole para producir bienes o prestar servicios. Los recursos materiales son predecibles, detectables o medibles pero el factor humano se presenta con matices únicos que son muy difíciles, sino imposibles de controlar.

Por ello, requiere muchos recursos encontrar a una persona cuyas cualidades se ajusten con precisión a los requerimientos de un puesto de trabajo. Y si agregamos los conocimientos, las habilidades, así como la experiencia y las actitudes que un trabajador debe tener para desarrollar productivamente el puesto que se le asigne en una organización.

Grados (2007) añade a la dificultad de encontrar un candidato idóneo lo siguiente:

(...) ya que sobre la organización actúan fuerzas impredecibles, como el perfeccionamiento de programas tecnológicos y de productividad, las características dinámicas del mercado económico en el cual actúan, la implantación de nuevas leyes, el volumen de operaciones que maneja y la inflación, que la obligan a efectuar cambios en sus objetivos, en sus sistemas, métodos y procedimientos. (p. 55)

Por ello, concluimos la imperiosa exigencia de la capacitación continua e integral para actualizar los conocimientos, desarrollar las habilidades y guiar las actitudes de los integrantes de la organización, con el fin de garantizar la supervivencia, crecimiento y adaptación institucional en un mercado en constante transformación.

En torno al aprendizaje y capacitación, Grados (2007) considera que existen tres dimensiones de desarrollo de las personas:

\section{Área cognoscitiva}

Son las competencias intelectuales que influyen en el desempeño de procesos que ha de realizar la persona. Incluye la memoria, el análisis, la abstracción, la atención y las estrategias de reflexión. Estos mecanismos permiten el conocimiento crítico de la realidad y las herramientas de interacción que debe dominar. Los programas de capacitación modifican las habilidades cognoscitivas y esto se puede acreditar en la medición de los conocimientos.

\section{Área psicomotriz}

Está constituida por las habilidades manuales y de coordinación sensorio motriz específicas. Incluye las rutinas y destrezas verbales, así como de movimientos y coordinaciones corporales que se requieren en la organización. La capacitación en la psicomotricidad incrementa estas habilidades traduciéndose en un aumento de la rapidez y efectividad de las actividades manuales de los colaboradores.

\section{Área afectiva}

Formada por las actitudes que se manifiestan conforme a la esfera valorativa y criterios del empleado. Generan impulsos básicos para actuar frente a las personas, los acontecimientos y las relaciones estructurales. Está de más subrayar que influyen en el desempeño del trabajo. Incluye la autopercepción y autoestima, así como los ideales, sentimientos, valores, emociones e inclinaciones únicas e íntimas que el individuo integra en la estructura de su personalidad. La capacitación en el área afectiva se expresa en el cambio de actitudes consigo mismo y los demás.

Los programas de capacitación tienen por finalidad lograr la potenciación de las competencias del personal porque:

- Logran que los empleados sean más productivos y estén preparados para la adaptación a las nuevas exigencias del puesto y del mercado.

- El éxito de la organización depende del desarrollo de los individuos que la integran. Esto supone la determinación de las necesidades de crecimiento individual y su satisfacción por medio de la capacitación y las oportunidades de desarrollo.

- Las personas que participan en las organizaciones quieren su propio desarrollo 
laboral, económico y social. El trabajo les proporciona la oportunidad de poner en marcha sus competencias. Al mismo tiempo, la organización se beneficia de los progresos individuales creándose un ambiente nutritivo para la empresa y sus colaboradores.

Cuando las organizaciones presentan dificultades se suele recurrir a consultorías en las áreas que lo requieran. Cuando las dificultades se presentan en las personas se requiere de una evaluación del ambiente laboral y de las necesidades de capacitación. Esta fase conlleva muchos retos. Es menester tener en cuenta que, si este proceso falla, la capacitación caerá en saco roto y los costos serán mayores. Por ello, debemos realizar este proceso con sumo cuidado y diligencia para obtener mejores resultados de la inversión.

\section{Capacitación eficiente.}

Una labor importante que deben asumir las autoridades de la UNMSM es identificar al personal que requiere de capacitación específica relacionada a las labores que el trabajador viene realizando. Pero las capacitaciones deben estar limitadas a las funciones requeridas en el puesto de trabajo. Todos los servidores docentes, administrativos y obreros gozan del derecho a recibir capacitación permanente y de calidad, pero no puede hablarse de una capacitación generalizada. Los programas de capacitación dependerán de los grados de instrucción y especialización que tengan los trabajadores, así como de las funciones que desarrollan, del tiempo de servicios, el vínculo laboral que mantienen con la UNMSM y otros.

Se requiere establecer las diferencias entre las competencias de los trabajadores y los objetivos institucionales para evitar, no sólo una repetición infértil de datos conocidos o la suposición de conocimientos existentes, sino una inversión inútil de los recursos institucionales.

El primer sesgo que debemos evitar para determinar inequívocamente las necesidades de capacitación, surge de los mismos trabajadores. Según Dowling (2005):

Cuando se les pregunta a los trabajadores si tienen las competencias necesarias para el puesto, no siempre se obtiene una respuesta veraz. Algunos ocultan su falta de conocimientos o comprensión por timidez, o temor. Otros creen que, si no responden afirmativamente, no obtendrán las oportunidades que desean. (p. 24)

Es ineludible evaluar las competencias de cada trabajador para que pueda llevar a cabo sus tareas y funciones en forma independiente, sin necesitar apoyarse en los conocimientos de sus compañeros.

Debemos tener presente que la capacitación del personal supone su planificación para hacerla eficiente y que requiere según Dowling (2005):

a. Evaluar constantemente el nivel de comprensión.

b. Adecuar el nivel de capacitación a los participantes.

c. Presentar un número limitado de conceptos a la vez. Por ello es que las capacitaciones, por lo general, se dan en sesiones (con el fin de que los participantes no se sientan abrumados con tanta información a la vez).

d. Involucrar a todos los trabajadores (que todos participen activamente).

e. Usar material visual. Sobre todo, ahora que se habla de la educación audiovisual, que facilita el proceso de conocimientos.

f. Estimular a los participantes para que formulen preguntas sobre el tema. Esto se hace para asegurar una retroalimentación adecuada.

\section{Capacitación como herramienta de la gestión organizacional.}

Tipos de capacitación.

Tomando en cuenta la taxonomía de Grados (2005) podemos establecer los siguientes tipos de capacitación:

\section{a. Capacitación interna}

La capacitación interna se puede realizar en dos formas: 


\section{a.1 Capacitación en centros internos}

La capacitación en centros internos es realizada por unidades orgánicas que forman parte de la institución son financiadas por ella en su integridad, aunque no necesariamente se utilizan los recursos humanos ni las instalaciones de la institución. Este concepto se refiere a que la capacitación y desarrollo del personal forma parte de las políticas institucionales.

Evidentemente, la capacitación del personal en instituciones estatales está en función de su finalidad pública y se adecua a los requerimientos organizacionales. Por ello, según Grados (2005) no se deben establecer patrones idénticos para todos los trabajadores de una misma institución ni pretender la evaluación de la capacitación con los mismos procedimientos ni con los mismos instrumentos.

Por lo general los programas de aprendizaje se realizan a través de métodos expositivos tales como cursos, clases, talleres o conferencias; en otras ocasiones excepcionales se requiere de programas de alta especialización.

\section{a.2 Capacitación en servicio}

Es una modalidad que merece un mayor interés por parte de las autoridades universitarias. Es notoria la desatención de esta forma de empoderamiento institucional por parte de los funcionarios de la alta dirección.

La capacitación en servicio tiene por finalidad preparar a un servidor para hacerlo apto para determinado cargo. Un instructor ad hoc o el superior inmediato es quien se encarga de todo o casi todo el entrenamiento.

Los objetivos de los programas de adiestramiento en servicio son, por lo general, adiestrar al personal nuevo en las funciones y tareas específicas del cargo que han asumido; capacitar al personal experimentado para la realización de funciones distintas a las requeridas en su puesto de trabajo o en puestos superiores al suyo; y, capacitar a los trabajadores de planta para crear una nómina de profesionales y técnicos de distintos niveles que se encuentren debidamente instruidos para enfrentar eficientemente las vicisitudes de la rotación de personal.
La implementación de programas de capacitación en servicio comienza con cursos teóricos y talleres acompañados de visitas a las instalaciones y diferentes dependencias institucionales. Esto permite una primera aproximación a las funciones a realizar y familiarizar al personal con las tareas futuras.

Los programas de capacitación en servicio son de duración variable; pero por lo general duran entre dos y tres meses. La capacitación muy especializada requiere de períodos mayores que oscilan entre 6 y 9 meses.

Según Grados (2005) las organizaciones que mantienen programas de capacitación internos, por lo general no evalúan el beneficio que obtienen del rendimiento del personal profesional que ha sido capacitado. Asimismo, la participación de los trabajadores en la capacitación sólo se premia con certificados o documentos análogos que confirman su asistencia o aprobación de un programa de capacitación. No establecen gradualidad en el logro de los objetivos de capacitación que motiven la competencia interna entre los trabajadores. Por lo general son pocos los participantes que obtienen calificaciones numéricas.

\section{b. Capacitación externa}

Este tipo de capacitación es programada y realizada por organizaciones o personal ajeno a la organización. Los responsables de capacitación son reclutados por los administradores de la institución con la finalidad de potenciar, según corresponda, las competencias afectivas, cognitivas o psicomotrices de los colaboradores para lograr la eficiencia esperada en sus actividades.

La experiencia y pericia laboral obtenida mediante capacitación externa siempre abona en favor de la mejora de la productividad interna, así como proporciona mejores oportunidades para la discusión con otras personas que enfrenten problemas de la misma naturaleza.

\section{Evaluación de programas de capacitación: su impacto sobre resultados.}

Tipos de evaluación de programas.

La evaluación integral de los programas de capacitación incluye varias formas, instrumentos 
y modalidades de determinación del éxito del programa.

La evaluación de los programas incluye las siguientes actividades de control concurrente y posterior, según Grados (2005):

\section{- Monitoreo de performance}

Se evalúa la funcionalidad de un programa; cuantifica el cumplimiento de los objetivos del aprendizaje. En este sentido, mide el éxito en la ejecución de las funciones o tareas del personal después de la capacitación. Para ello, investiga si la persona capacitada realiza sus actividades con mayor eficiencia, si sus ingresos personales han mejorado, si comete menos errores, si presenta alternativas innovadoras, en fin, si se observa una mejora sustancial en su rendimiento laboral.

- Evaluaciones de impacto: se enfocan en la causalidad.

Supone una comparación del estado de la productividad entre el estado anterior y posterior a la capacitación. Mediante este procedimiento podemos reconocer el impacto de la capacitación. Para ello, se requiere de un estudio previo de las condiciones antes de la capacitación y después de la capacitación. Las diferencias temporales indicarán si los objetivos se alcanzaron o no.

- Evaluaciones de costo y costo-beneficio:

Se mide la relación entre los costos de la capacitación y el costo de otros medios utilizados con el mismo fin: mejorar la productividad. Con ello logramos establecer los beneficios que se han obtenido en ambos casos. Al comparar los resultados de ambos casos se podrá evaluar objetivamente si la capacitación ha sido más provechosa.

- Evaluaciones de procesos:

Este es una evaluación concurrente, permite el seguimiento del proceso de capacitación mientras se está realizando. Se puede aplicar encuestas o entrevistar a los participantes sobre la calidad de la capacitación y el impacto que está teniendo en los asistentes.

\section{Medición de resultados de una capacitación.}

La capacitación trae consigo un proceso de cambios que se pueden medir y, por ello, evaluar. Evidentemente los resultados de estos programas estarán vinculados al desempeño laboral del capacitado. Según Blake (2005) por lo general las organizaciones no han desarrollado mecanismos de evaluación de los resultados de la capacitación proporcionada a su personal.

La evaluación de los resultados de la actividad de capacitación se realiza estableciendo la diferencia perceptible en la ejecución de una misma tarea realizada en dos momentos distintos, pero en condiciones comparables. Este estudio longitudinal es aclarado por Mc Gehee (1973) cuando afirma que debemos diferenciar la simple evaluación de los resultados cognitivos, actitudinales o psicomotrices de la capacitación, del impacto que estas competencias han tenido sobre el desempeño laboral del personal capacitado. Es decir, medir el resultado de la capacitación en función de las destrezas logradas en la eficiencia del trabajo desempeñado.

Debemos recordar que cada actividad de la organización tiene su modelo de evaluación de resultados. Por ello, la evaluación de la capacitación puede diferir, y de hecho lo hace con mucha frecuencia tanto en instrumentos como en criterios, de los modelos de evaluación de otras áreas de la empresa.

\section{Evaluación y seguimiento de la capacitación.}

Siendo un proceso continuo y permanente, la capacitación del personal requiere de una evaluación y retroalimentación constante. Los mecanismos de control de calidad son inherentes al proceso de capacitación.

El valor más importante de las organizaciones o personas naturales que se dedican a la consultoría o capacitación es la confianza. Esta certeza del cliente, de que la calidad del servicio es alta va a permitir la fidelización del usuario del servicio. Para ello, el éxito o no de un programa de evaluación se refleja en el incremento de la productividad del trabajador. Mc Gehee (1973) indica que: 
Ugarte Casafranca et al.

Además del establecimiento de prácticas, éstas deben ser monitoreadas introduciendo tácticas de control de calidad. Para evaluar la capacitación, el capacitador debe vigilar la realización de tres tácticas control de calidad de la capacitación, las cuales son inspección, revisión y evaluación de la capacitación.

La inspección está diseñada para mantener el correcto desarrollo del programa de capacitación. En este sentido, según lo afirmado por Mc Gehee (1973) debemos centrarnos en la evaluación de la aplicación in situ de las competencias adquiridas producto del programa de capacitación más que en la fiscalización del programa en sí.

La manera más adecuada de realizar mecanismos de control es teniendo presente que la única forma válida de evaluar la capacitación es en función de los resultados. Por tanto, es necesario antes de iniciar la capacitación, debemos evaluar las competencias del personal, luego desarrollaremos un programa de adiestramiento y lo ejecutamos. Por último, evaluaremos si la capacitación ha tenido o no efecto en la productividad de los capacitados.

\section{Formas de evaluación.}

\section{Pre-prueba.}

Consiste en determinar las competencias y habilidades que tiene el personal sobre los aspectos que deseamos potenciar: atención al cliente, motivación, informática u otros. Este diagnóstico permitirá al capacitador redireccionar el programa de capacitación en función de las necesidades específicas de quienes han de ser capacitados. Este momento de la evaluación nos brinda la oportunidad de cambiar los contenidos, estrategias y el grado de profundidad de la capacitación. Por lo general, esta forma de evaluación se presenta en las modalidades siguientes:

\section{a. Escrita.}

Puede realizarse la aplicación de un cuestionario, solicitarles que escriban una breve monografía o estudio relativo a la competencia a evaluarse; o, en su defecto solicitarles que presenten alternativas para resolver un problema hipotético.

\section{b. Verbal.}

Puede usarse la entrevista libre o estandarizada, pero es posible obtener resultados más fructíferos si el personal relata personalmente o a través de medios audiovisuales cuáles son sus competencias, conocimientos o habilidades relacionadas al aspecto que se quiere evaluar. Es importante tener presente que las entrevistas logren que el trabajador pueda expresarse con naturalidad y espontaneidad.

\section{c. Práctica}

Esta evaluación requiere que los participantes realicen un conjunto de acciones encaminadas al logro de un objetivo previsto de antemano. Se medirá tanto los resultados como el tiempo de implementación de la acción.

Los instrumentos de evaluación deben arrojar datos cuantitativos. Sólo de esta forma podremos tomar decisiones objetivas sobre las necesidades de capacitación y sobre el nivel de conocimientos o competencias generales y específicas de los trabajadores.

\section{Evaluación del proceso de capacitación.}

Esta evaluación se realiza durante el proceso de capacitación y sirve para detectar el nivel de conocimiento que están alcanzando los capacitadores; además, permite volver a ajustar el contenido temático, el ritmo con que se desarrollan los temas y el clima que se está logrando en el grupo. En este sentido, Reza (1995) afirma:

En ocasiones advertimos que es necesario repetir un tema, aclarar dudas, proporcionar más ejemplos, modificar el tono de voz, o bien, cambiar de técnicas. También indica la velocidad con la que el grupo ha captado la información, lo que brinda oportunidad de profundizar en los temas o ampliar el temario. (p. 99)

\section{MÉTODOS}

El diseño de la investigación es exploratorio, descriptivo y no-experimental. Se enmarca dentro del tipo de investigación aplicada. La metodología empleada es la combinación de métodos de investigación: Inductivo-deductivo, analítico-sintético. 
Determinación de Necesidades de Capacitación del Personal Administrativo del Área de Ciencias Económicas y de la Gestión...

Hemos realizado entrevistas que nos permitieron contar con información real acerca del nivel de implementación de la Ley Servir, en el marco de la presente investigación y poder plantear la propuesta de un programa de capacitación del personal administrativo que coadyuvará al mejoramiento de los procesos internos de las facultades sometidas al estudio.

Las entrevistas fueron a funcionarios con cargos de jefatura que tienen personal subordinado. Las áreas estudiadas de las tres facultades fueron: direcciones administrativas, escuelas profesionales, direcciones de departamento, unidades de posgrado y unidades de matrícula.

Hemos aplicado una encuesta para recabar datos acerca de las necesidades de capacitación las cuales han sido tabuladas y analizadas con el fin de probar las hipótesis planteadas, y lograr los objetivos propuestos.

Según lo estipulado en el Artículo 42 del Estatuto de la UNMSM, se establecen las siguientes cinco (5) áreas académico-profesionales: Ciencias de la Salud, Ciencias Básicas, Ingenierías, Ciencias Económicas y de la Gestión, Humanidades y Ciencias Jurídicas y Sociales.

El área de Ciencias Económicas y de la Gestión comprende a la Facultad de Ciencias Administrativas, la Facultad de Ciencias Contables y la Facultad de Ciencias Económicas.

El personal administrativo de las tres facultades está constituido, como se muestra en la tabla 01.

Es notorio que la cantidad de trabajadores contratados bajo la modalidad del régimen especial de contratación administrativa (CAS) supera en más del doble la de personal nombrado. El total de trabajadores administrativos del área estudiada es 157.

\section{Selección de muestra}

Para obtener la muestra poblacional hemos aplicado la siguiente fórmula:

Muestra poblacional (n):

$\mathrm{n}=\frac{N(Z)^{2} P Q}{(E)^{2}(N-I)+Z^{2} P Q}=\frac{157 *(I 96)^{2} \cdot(5 \theta)(50)}{(I \theta)^{2} *(I 57-I)+1.9 \sigma^{2}(50)(5 \theta)}$

Donde:

$\mathrm{N}$ = Población

$\mathrm{Z}$ = nivel de confianza

$\mathrm{P}=$ probabilidad de éxito, o proporción esperada

$\mathrm{Q}=$ probabilidad de fracaso

$\mathrm{E}=$ precisión (error máximo admisible en términos de proporción)

\section{$\mathrm{n}=\frac{N(Z)^{2} P Q}{(E)^{2}(N-I)+Z^{2} P Q}=\frac{157 *(I 90)^{2} \cdot(50)(50)}{(I 0)^{2} *(I 57-I)+1.9 \sigma^{2}(50)(50)}$}

La muestra poblacional es de 60 personas, aplicando el redondeo. Sin embargo, hemos aplicado el instrumento en total a 89 colaboradores distribuidos según la población de cada facultad de la siguiente manera: Ciencias Administrativas (35), Ciencias Contables (28) y Ciencias Económicas (26).

El muestreo ha sido no probabilístico por convención (Alma, Méndez y Sandoval, 2011), porque se ha buscado a las personas conocedoras sobre el tema estudiado, así se han obtenido mejores informaciones y datos relevantes para la presente investigación. La encuesta ha sido tomada en base a lo estipulado por SERVIR, la cual tiene como finalidad conocer las necesidades de los colaboradores en relación a los indicadores sobre el perfil del puesto en apoyo a la gestión y uso de la tecnología de la información.

Tabla 01.

Personal Administrativo por facultad

\begin{tabular}{lcccccccc} 
& & \multicolumn{2}{c}{ Sexo } & & & & & \\
Facultad & Total & & H & M & Nombrado & Contratado & Designado & CAS \\
\cline { 3 - 4 } Ciencias Administrativas & 61 & 32 & 29 & 14 & 2 & 4 & 41 \\
Ciencias Contables & 50 & 17 & 33 & 12 & - & 5 & 33 \\
Ciencias Económicas & 46 & 21 & 25 & 26 & 2 & 1 & 17 & 91 \\
Total & 157 & 70 & 87 & 52 & 4 & 10 & 91 \\
\hline
\end{tabular}

Nota. Elaboración propia. Adaptada del Compendio Estadístico - UNMSM - 2018. Recuperado de Compendio Estadístico 2019. 
La escala usada ha sido (1) menos importante, (2) medio importante, (3) importante y (4) más importante. Para medir, se usaron dos preguntas centrales, la primera pregunta centrada en la capacitación que requiere mejorar en el desempeño de acuerdo al perfil de puesto con 27 cursos; y la segunda pregunta central centrada en la capacitación para mejorar el desempeño en materia de informática compuesto de 10 cursos.

\section{Validación del instrumento}

La validez del instrumento de investigación se realizó por la prueba de criterio de jueces, para este proceso se invitó a cinco jueces, entre ellos al Dr. José Villacorta Huapaya, Dr. Jorge Mayuri Barrón, Mg. Carlos Zúñiga Reynoso, Dr. Walter Alva Miguel y el Dr. Edwar Espinoza Herrera; los cuales mostraron interés de realizar la evaluación del instrumento de investigación, y se procedió a entregarles los documentos de evaluación vía virtual; los resultados fueron enviados al correo del investigador principal. Cuando se obtuvieron los datos se organizaron y se aplicó el coeficiente $\mathrm{V}$ de Aiken. Los resultados muestran la validez de las alternativas (ver tabla 2 ).

\section{Objetivos}

\section{Objetivo general.}

Determinar el grado de implementación de los programas de capacitación de personal, en el marco de la Ley 30050 Ley de Servicio Civil, en las facultades de ciencias económicas de gestión de la Universidad Nacional Mayor de San Marcos.

\section{Objetivos específicos.}

\section{Objetivo específico 1.}

Elaborar la descripción de puestos administrativos de las facultades de ciencias económicas y de la gestión de la UNMSM

\section{Objetivo específico 2.}

Determinar las necesidades de capacitación del personal administrativo de las facultades de ciencias económicas y de la gestión de la UNMSM

\section{RESULTADOS}

\section{Datos}

La mayoría de encuestados considera la necesidad de capacitación en habilidades blandas en especial en trabajo en equipo, seguidos del manejo de conflictos y liderazgo. (Ver Figura 1)

La mayoría de encuestados considera necesaria la capacitación de informática en Excel, PowerPoint y Access, seguido en menor proporción de diseño de curso en línea. En menor importancia consideran el manejo de videoconferencia y elaboración de páginas web. (Ver Figura 2)

La mayoría de encuestados considera necesidad de capacitación en gestión administrativa sobre todo en manuales de procedimientos, manuales administrativos, seguidos de los manuales de organización y control de calidad. En menor importancia consideran el control de gestión y orientación a resultados. (Ver Figura 3)

El 75\% de los encuestados consideran de mayor relevancia la capacitación en habilidades blandas, especialmente en trabajo de equipo (56\%) y manejo de conflictos (19\%). (Ver Figura 4)

\section{DISCUSIÓN}

Uno de los retos más importantes de las autoridades de la UNMSM es obtener información clara, precisa y confiable del personal administrativo que requiere un tipo de capacitación específica, relacionado con las funciones y tareas

Tabla 02.

Validez de alternativas según expertos

\begin{tabular}{llcccccccc}
$\mathbf{N}^{\circ}$ & & Juez 1 & Juez 2 & Juez 3 & Juez 4 & Juez 5 & Total & V de Aiken \\
1 & Cursos de capacitación de desempeño según perfil del puesto & 1.00 & 1.00 & 1.00 & 1.00 & 1.00 & 5 & 1.00 & \\
& Cursos & 1.00 & 1.00 & 1.00 & 1.00 & 1.00 & 5 & 1.00 & \\
2 & Cursos de capacitación de desempeño en informática & 1.00 & 1.00 & 1.00 & 1.00 & 1.00 & 5 & 1.00 & 1.00 \\
\hline
\end{tabular}

Nota. Los resultados del coeficiente $\mathrm{V}$ de Aiken indican la validez de las alternativas de la encuesta aplicada en el estudio. Elaboración propia. 
Determinación de Necesidades de Capacitación del Personal Administrativo del Área de Ciencias Económicas y de la Gestión...

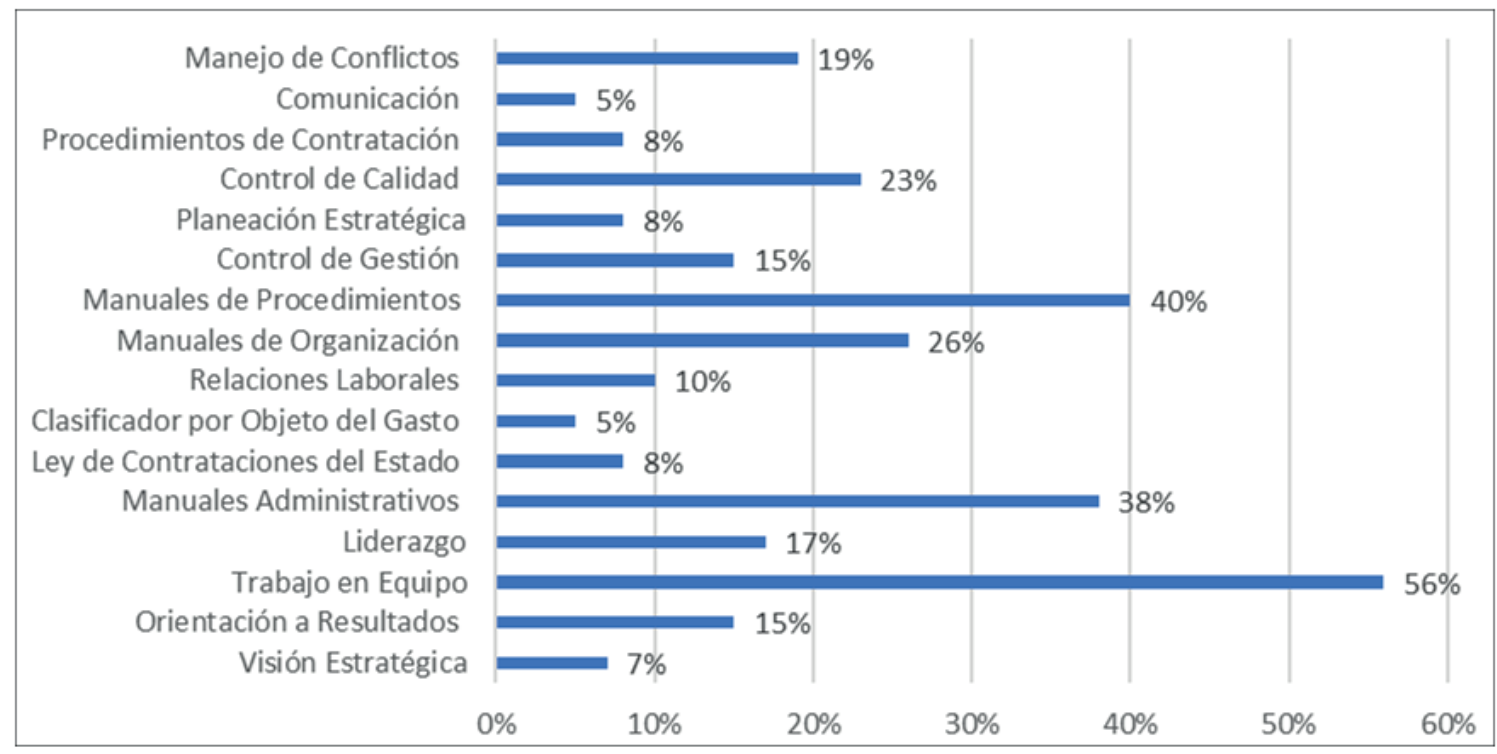

Figura 1. Necesidades de Capacitación en Porcentajes. Elaboración propia.

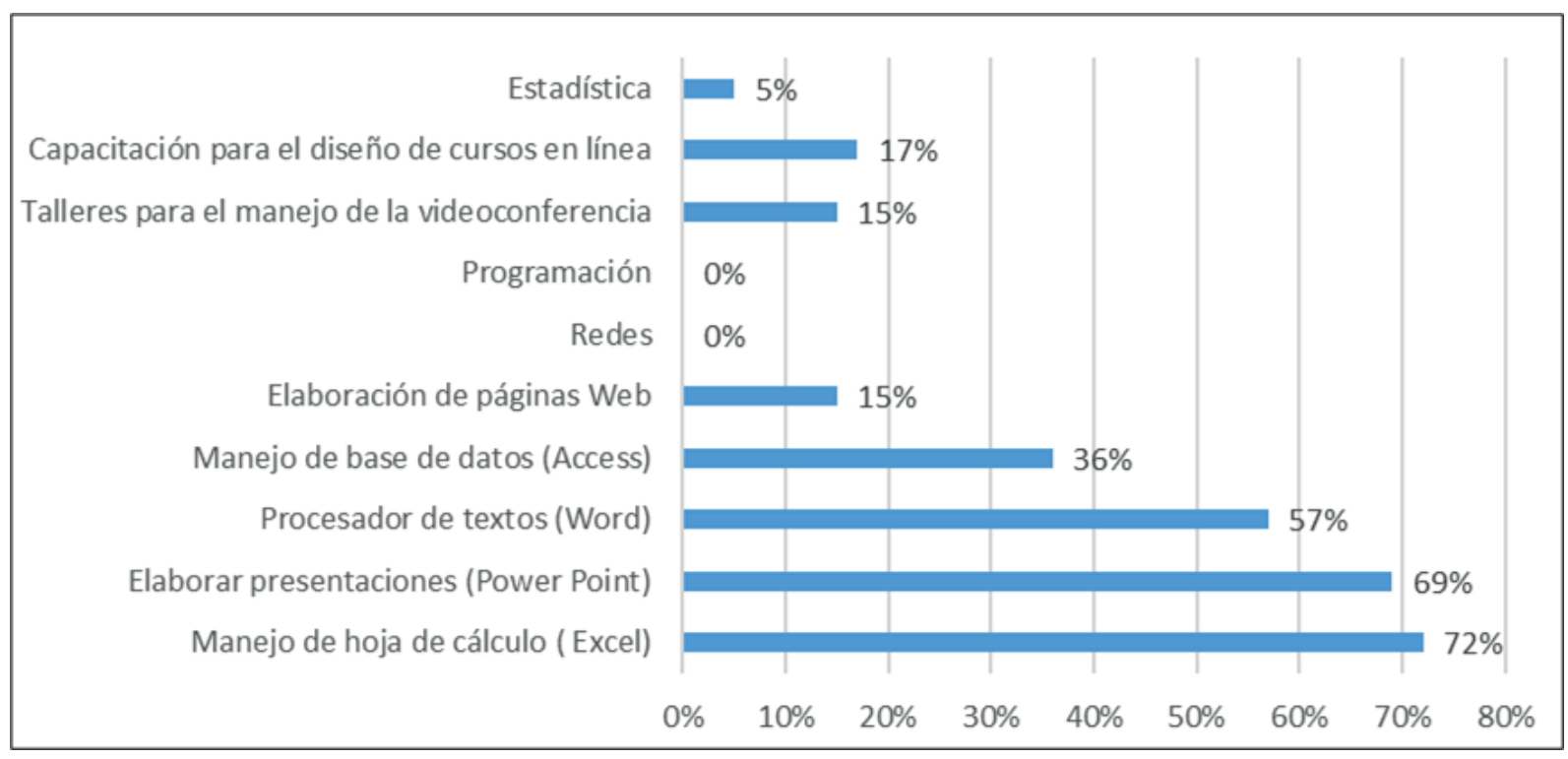

Figura 2. Necesidades de capacitación en informática en porcentajes. Elaboración propia.

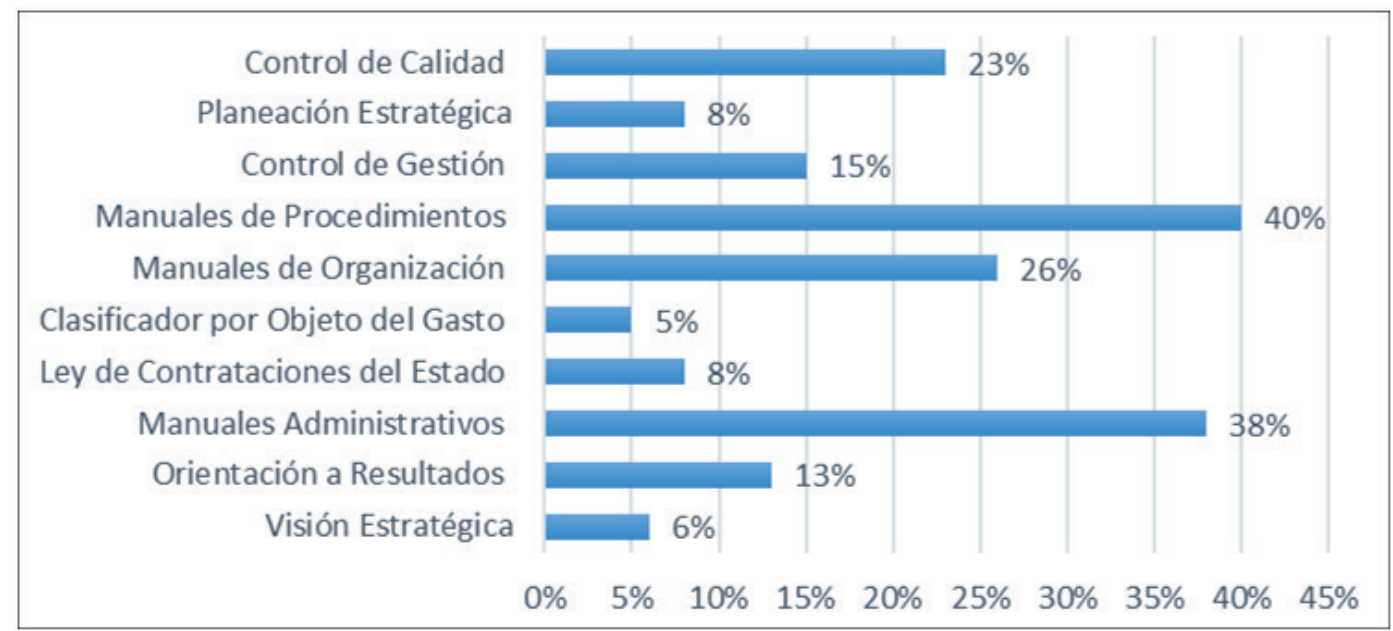

Figura 3. Necesidades de capacitación en gestión administrativa en porcentaje. Elaboración propia. 


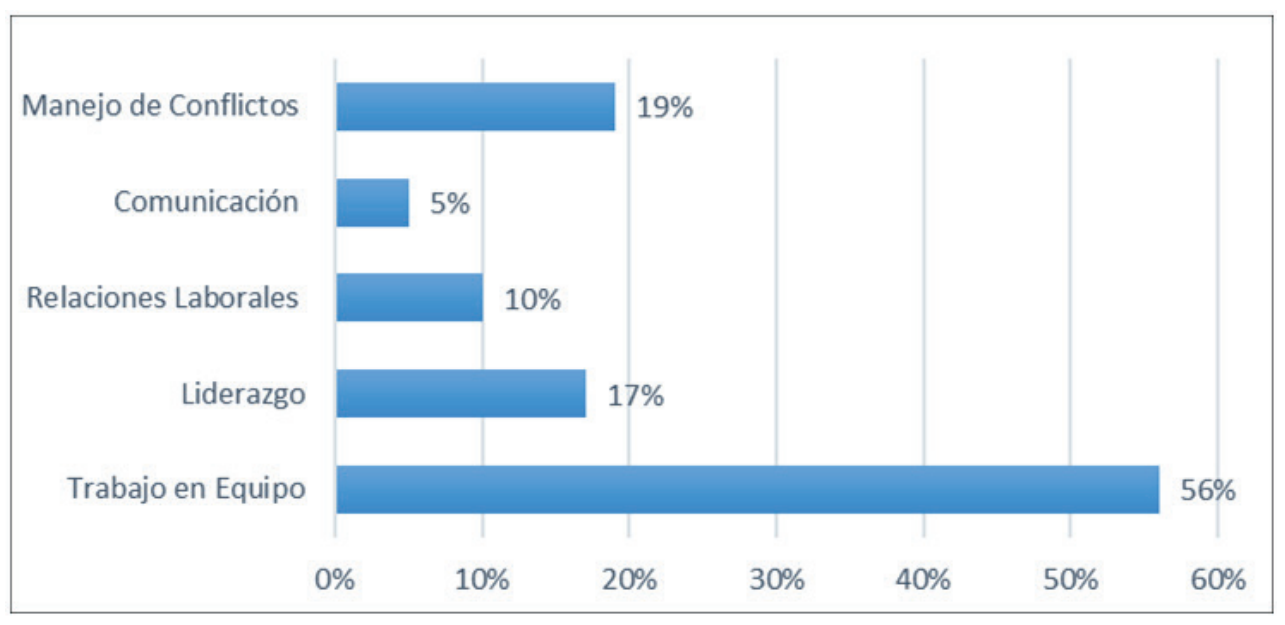

Figura 4. Necesidades de capacitación en habilidades blandas. Elaboración propia.

de los puestos que ocupa. Sin embargo, debemos tener presente que la capacitación integral no se limita únicamente a las actividades cotidianas desempeñadas en el puesto de trabajo. Siendo una institución universitaria, se requiere de todo el talento de los servidores a fin de cumplir con la finalidad pública de nuestra institución. El contacto constante entre el personal administrativo con el resto de la comunidad universitaria hace necesario que el talento humano a desplegarse vaya más allá de la mecanización de procedimientos y la aplicación técnica de las normas. Existe un fuerte componente humano en el cual las competencias afectivas juegan un papel preponderante. No basta que el trabajador simplemente "cumpla su trabajo" sino que lo haga de manera tolerante, humana y con dignidad.

Por ello, según Dowling (2005):

cuando se les pregunta a los trabajadores si tienen las competencias necesarias para el puesto, no siempre se obtiene una respuesta veraz. Algunos ocultan su falta de conocimientos o comprensión por timidez, o temor. Otros creen que si no responden afirmativamente, no obtendrán las oportunidades que desean. (p. 24)

Por ello, la planificación de la capacitación requiere de una evaluación previa de las capacidades de cada servidor para que pueda desempeñarse en forma independiente. Claro está que además de las competencias individuales, debemos dar prioridad al trabajo de equipo.
Según la data que hemos obtenido a partir de entrevistas y encuestas, una gran proporción de los trabajadores considera que se debería capacitar para mejorar las relaciones interpersonales y potenciar las habilidades sociales en la solución de conflictos laborales. Esto nos indica que los conflictos interpersonales son un lugar común en las relaciones institucionales.

Asimismo, es notorio un desconocimiento de las normas administrativas. Esta carencia tiene como consecuencia el retraso constante de los trámites de la gestión en general. Siendo una institución que desarrolla sus actividades con escasos recursos no podemos darnos el lujo de invertir demasiado tiempo en tramitología que demora la ejecución de los proyectos y en el cumplimiento de los compromisos. Es constante la queja, tanto de proveedores externos, del personal docente, así como del estudiantado en relación al lapso excesivo que se toma en la finalización de los procedimientos.

Uno de los puntos más resaltantes es la atomización de los esfuerzos. Esto debido a la falta de liderazgo. Por ello, la mayoría de los trabajadores, conscientes de ello, consideran que se debe hacer hincapié en la capacitación sobre trabajo en equipo.

\section{CONCLUSIONES}

1. La gestión de la capacitación en las facultades del área económico empresarial carecen de metodología y programación. 
2. El presupuesto asignado a la capacitación del personal administrativo de las facultades integrantes del área económico empresarial es insuficiente.

3. Los trabajadores consideran muy importante el desarrollo de capacitación en el perfil personal de los puestos.

4. Se requiere programar capacitación para el desarrollo de las competencias blandas.

\section{REFERENCIAS BIBLIOGRÁFICAS}

Alva, R. (2014). Las tecnologías de información y comunicación como instrumentos eficaces en la capacitación del personal: El caso de la Oficina Nacional de Procesos Electorales (ONPE) (Tesis de maestría). Universidad Nacional Mayor de San Marcos, Lima, Perú.

Dowling, J. (2005). Cómo desarrollar un programa de capacitación. México: Pearson.

Grados, J. (2007). Capacitación y Desarrollo Personal. Trillas, México.
Medina, F. (2014). Diseño del plan de capacitaciones en base al modelo de retorno de inversión - ROI - para la planta central del Ministerio de Defensa Nacional, correspondiente al período fiscal 2013 (Tesis de maestría). Pontificia Universidad Católica del Ecuador, Quito, Ecuador. recuperado de http://repositorio.puce.edu.ec/ bitstream/handle/22000/8130/TESIS $\% 20$ FINAL\% 20NOVIEMBRE\%20INCLUYE \% 20 HOJA.pdf?sequence $=2$

Mc Gehee, W. (1973). Capacitación: Adiestramiento y Formación Profesional. Limusa, México.

Mustaffá, J. (1976). Capacitación de Personal. Alide, Lima.

Reza, J. (1995). El ABC del Administrador de la Capacitación. México: Panorama Editorial.

Segovia, R. (2013). Gestión del conocimiento en una Entidad Pública a través del uso de plataformas virtuales de enseñanza: caso defensoría del pueblo (Tesis de maestría). Pontificia Universidad Católica del Perú, Lima, Perú. 
